\title{
Strategic use of Representation in Architectural Massing
}

Omer Akin: Carnegie Mellon University: oa04@andrew.cmu.edu

Hoda Moustapha: Carnegie Mellon University: hoda@ andrew.cmu.edu

Keywords: architectural massing, design strategies, protocol analysis, regulating elements.

\begin{abstract}
Architectural massing is the primary subset of the early stages of built form creation. In this empirical study, we set out to better understand the specific cognitive processes contributing to massing. We found that these processes help the designer better manage the overall design process through strategies that facilitate a discourse between designer and her graphic representations. These strategies, which rely on the use of regulating elements, include management of part-whole relationships, design hierarchy, topologygeometry relationships, scaffolding the design process, structuring ill-structured problems, and the restructuring of problem parameters.
\end{abstract}


Architectural massing is the act of composing and manipulating three-dimensional forms into a unified, coherent architectural configuration. During this process, the relations among massing elements are studied; this includes the relations of the building with its surrounding context and of the building with its subparts. Massing comprises all decisions affecting external architectural form. It is a crucial component of design because it is the phase where a designer defines her building's identity as well as the impact of her building within its urban environment.

Le Corbusier defined architecture as "the masterly, correct and magnificent play of masses brought together in light" In his statement, there is an emphasis on massing as if it were the very essence of Architecture ${ }^{1}$.

Architectural massing is mostly described as a product in architectural design literature, and has not been sufficiently analyzed as a process. Therefore, we set out to analyze the cognitive strategies that contribute to architectural massing. Our primary motivation was the designing of effective computational tools that would support massing. Although the study presented in this paper focuses on cognitive aspects of massing, it is a part of a larger research effort dealing with the computational aspects required to support massing. These will be discussed briefly in the Discussion Section.

Our initial study consisted of observing six architects while they were performing early design activities that lead to three-dimensional massing models. We collected protocol data that revealed specific mechanisms that were repeatedly and consistently used as an integral part of several widely recognized cognitive strategies. These include management of part-whole relationships, design hierarchy, topologygeometry relationships, scaffolding the design process, structuring ill-structured problems, and the restructuring of problem parameters.

We begin our discussion with a description of our empirical study followed by a review regulating elements used as design strategies. Then, we discuss each strategy as described in literature and manifested in our protocol episodes. We conclude by discussing concepts for computational support for massing strategies.

\section{Empirical Observation}

Our initial investigation of the field revealed that the cognitive aspects of massing have not been sufficiently described in architectural or design process literature. Consequently we set up an empirical study for the purpose of gaining a deeper understanding of the strategies that guide the massing process. Since massing does not occur in isolation, we designed a series of protocol sessions that capture massing as well as early design activities that may be peripheral, while influencing massing decisions; for instance, the site design, layout planning, or elevation design.

\subsection{Experimental Set-up}

Protocol analysis has been widely used for studying human cognitive behavior within problem-solving contexts $^{2}$. Advantages and disadvantages of protocol analysis have been debated extensively ${ }^{3}$ and are outside of the scope of this paper. We chose protocol analysis because it captures comprehensively and simultaneously the designer's graphical representation and her corresponding verbalization. These verbal expressions encapsulate the motivation and rationale that are not evident in graphical representations therefore, clarifying possible ambiguities that are often present in the latter.

Our protocol experiment consisted of observing six architects while designing a three-dimensional massing model. Each session lasted two hours on the average, but was not limited by time, in order to avoid putting participants under pressure. Each session was recorded on videotape and was followed by a brief postexperiment questionnaire. The instructions and the questionnaire are in appendix 1 and 2, respectively.

We considered all participants as professional architects. Each had completed at least five years of architectural design education, and had variable work experience ranging between two to twenty-five years. Among the six participants, three were experienced CAD users with a minimum of three years experience. 
The task consisted of designing the three-dimensional massing configuration of a dormitory building on the Carnegie Mellon campus. The program included various functions, both repetitive and specialized, with different privacy levels (Appendix 3). The site was fairly complex, with a steep slope and two axes of orientation (Appendix 4). The required outcome was a 3D model; plans, sections, and elevations were optional. Due to its level of complexity, the chosen task had potential for uncovering interesting design strategies and a variety of solutions within a limited time frame.

Three sessions were completed in the sketch medium (pencil and paper) and three in the computing medium (CAD system). This allowed us to capture strategies that transcend representational media. In the computing sessions, three different industry-standard CAD systems were used (AutoCAD, MicroStation, and FormZ) so that the results would not be influenced by the limitations of a single system. The participants were also allowed to use paper whenever they needed in order to reveal the massing design activities that are difficult to achieve on the computer. Furthermore, the switch between the media is considered to be significant data for analysis in other studies. ${ }^{4}$

\subsection{Analysis}

We refer to participants as P1 to P6 and the corresponding protocol sessions as S1 to S6. The first three sessions ( $\mathrm{S} 1$ to $\mathrm{S} 3$ ) used the pencil/paper medium, while the remaining sessions used a CAD system. Each participant used a different one of the three available systems. Alternatives generated by each participant are referred to as Ail to Ain, where 'i' corresponds to the participant ID.

The protocol sessions were first transcribed into a series of moves, which included verbal expressions, drawing actions or their combination. Then these moves were categorized and color-coded according to the activity to which they contributed (massing, planning, site analysis, program review, design structure) and their type (analysis, decision, and evaluation). Moves are referred to as Mi_n.n.n, where ' $\mathrm{i}$ ' is the participant ID and n.n.n is the move number. The vocabulary of massing elements, precise massing manipulations, and massing strategies were identified in the transcriptions. Episodes illustrating substantial massing strategies were further analyzed.

\subsection{Summary of results}

The principal mechanism used in structuring massing activities was the use of regulating elements. These include tools like points of intersection, centers of rotation, lines and planes of intersection, axes of rotation, axes of symmetry, alignment, diagonal proportion lines, and bounding lines, planes and volumes. All participants maintained geometric order in their designs using such mechanisms. Although, they freely manipulated, added and removed massing elements, through the use of regulating elements they preserved their underlying structure and even accentuate them.

Analysis of the data showed that designers used these massing strategies, among other things, to regulate the overall design process. They used these strategies to help them manage part-whole relationships, design hierarchy, topology-geometry relationships, design propagation, structuring sub-problems, and the restructuring of problem parameters. In the following sections, we will consider each of these strategies as described in the literature and manifested in our massing episodes. We believe that these strategies illustrate that representation is used not as a passive mechanism merely to display the content of the mind onto external media, but to actively guide design.

\section{Regulating elements}

In each massing configurations, there is an underlying structure. ${ }^{5}$ It is common practice in architecture to express the relational structure of massing elements through regulating lines. As a matter of fact, in the past prominent architects have actively promoted the use of regulating lines. According to le Corbusier, "A regulating line is an inevitable element of Architecture ... It is an assurance against capriciousness.... it confers on the work the quality of rhythm.... The regulating line is a satisfaction of a spiritual order, which 
leads to the pursuit of ingenious and harmonious relations.... The choice of regulating line fixes the fundamental geometry of the work". ${ }^{6}$ JNL Durand, in an effort to systemize the architectural design process, introduced a "method to follow in the composition of any project", which relies on establishing the structure though regulating lines as a primary design step. ${ }^{7}$ Both Le Corbusier's and Durand's prescriptive approaches highlight the role that regulating lines have played in the development of architectural theory.

We extend the concept of a regulating line to a regulating "element" to include points, planes and volumes, which, in combination, express the entire structure of the architectural configurations in three dimensions. Regulating elements relate the massing configuration with surrounding buildings or other significant structures; they organize and structure the elements of massing configurations; form the spatial subdivision within masses; and relate massing elements to their constituents.

Our concern in this study is not whether or not designers use or should use regulating lines, but rather "how" they strategically use them in the creation of architectural form and the development of a design.

\section{Regulating the design process}

A method par excellence of the designer is "divide and conquer." ${ }^{8}$ Design problems are too large and complex. Finding a design solution that satisfies all aspects of a problem invariably requires that the problem be decomposed into sub problems. Once they are in hand, the solutions to each sub-problem needs to be re-assembled into a comprehensive solution. Specific strategies used by expert designers to synthesize partial solutions have been codified by Akin attesting to the important role that decompositionrecomposition strategies play. ${ }^{9}$

During the design sessions we studied here, participants were observed using regulating elements of massing to organize their work. A popular regulating element is the symmetry or alignment line that aligns individual design elements (whether they are rooms, windows, columns or stairs) with respect to it. Aside from the compositional orders that result from such use, symmetry or alignment axes represent metaelements that control the spatial organization of other, lower level elements. The relationship between the latter to the former is one of part to whole. For example, a room arranged with respect to an axis is a mere component of a larger composition defined by the axis and the colony of rooms.

\subsection{Management and of part whole relationships}

In our protocol study, we frequently observed the use of regulating axes. Aside from the formal role that they serve, these axes provide the key element for relating the parts to the wholes.

Most cognitive design process literature considers external representation as a means for making records of mental concepts. ${ }^{10}$ Often the focus is to identify, enumerate, and relate design moves in an attempt to model cognitive design processes. However, some sources such as Suwa and Tversky, believe that drawings reveal not only designer's thinking, but also they facilitate constraint inference problem solving and understanding. ${ }^{11}$ Van Summers explains that drawing can be a production system that helps people generate concepts. ${ }^{12}$ Goldschmidt describes visual thinking through interactive imagery as the two-way interaction between the mental concepts and sketch representations. Her evidence illustrates that designers sketch to help generate new images and ideas in their mind, in addition to externalizing ideas and images. ${ }^{13}$

In our protocol sessions, the participants used external representations, whether verbalizations, sketches, or computational records, with the apparent purpose of carry on a design dialogue with themselves. We would like to focus our attention on specific representation mechanisms that they strategically choose not only to generate ideas, but also to guide further design decisions and to structure upcoming design developments. They used regulating elements to express their design organization, either explicitly or implicitly. The explicit expression took either a graphic or verbal form. In the implicit expression, the relation was defined by the position of elements, but no lines were drawn nor discussed. Below we provide examples to support each of these forms of using axes to organize design elements. 


\subsubsection{Explicit-graphic}

In S3, the participant repeatedly draws the main axis of the configuration on every trace over the design sketch. This is substantiated at several points in the design protocol, one of which is illustrated in Episode 1 , below.
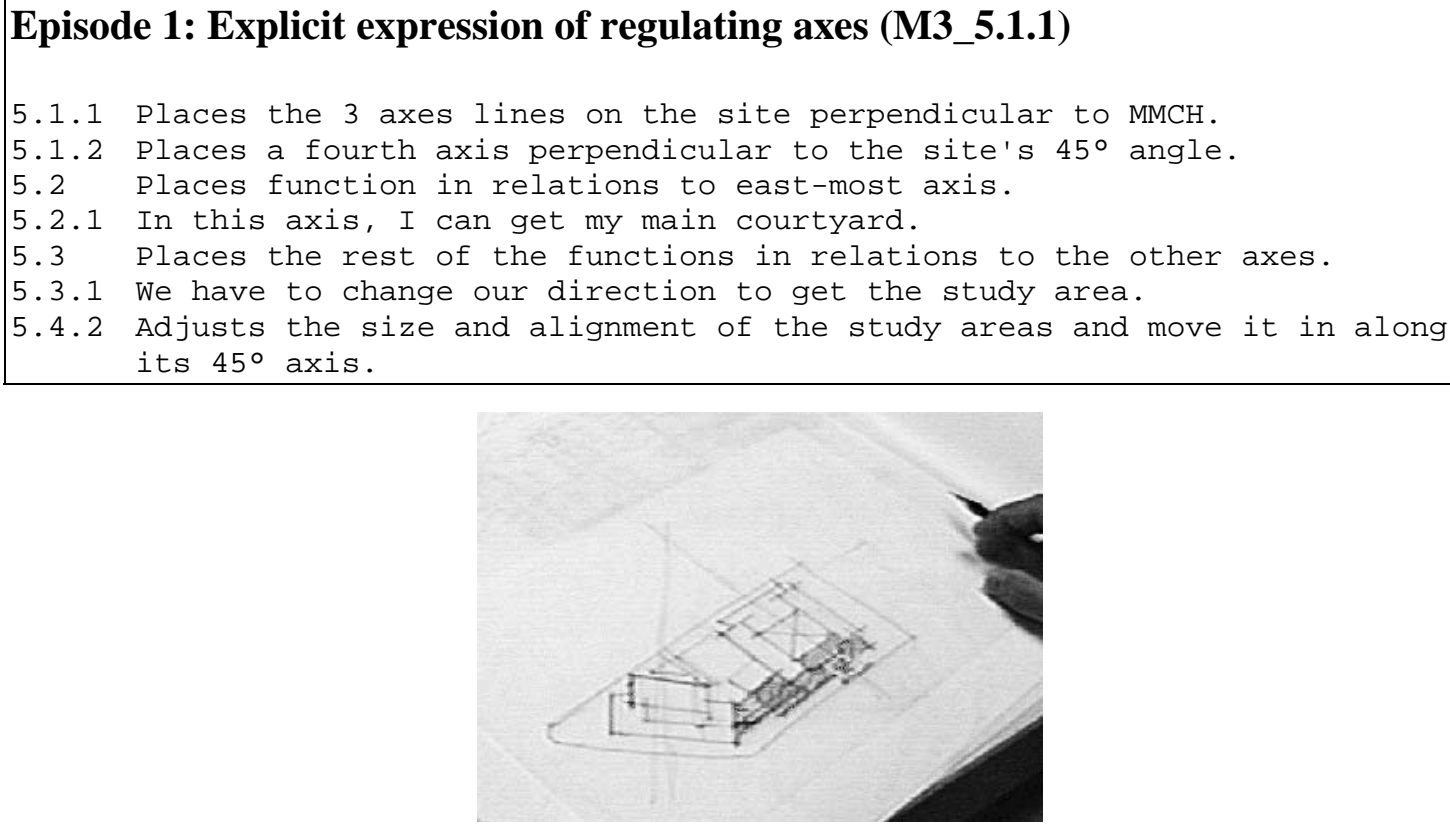

The axis is clearly the organizing element for the courtyard the two room-wings of the building and the restaurant. These parts are used to create the whole (the entire building) by virtue of the axis, without which the parts remain a set of unrelated elements.

\subsubsection{Explicit-verbal}

In $\mathrm{S} 4$, the participant defines the geometric structure verbally prior to any drawing activity. Even though the axis is not explicitly depicted, its verbal presence serves, just as successfully, the same function that the explicit axes serve in other protocol episodes (Episode 2). In the example below, we see the evidence that supports the contention that verbally stated axes are just as functional as the others.

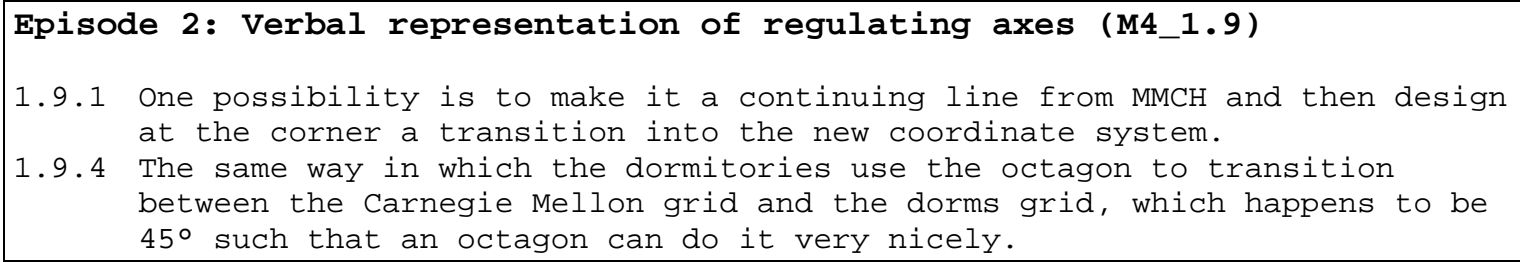

\subsubsection{Implicit}

The power of the axis as a design strategy is perhaps best expressed when it is used implicitly as a virtual line. This clearly suggests that the designer is not relying on the axis line as a compositional element in itself. Rather its sole function seems to be one of regulating the relationship between design elements and the meta-organizational concept of symmetry. Implicit axes derived from other elements in the design, can also be considered as emergent structures. 
The participant P2, for example, sets out to design an axially bilateral, symmetric building. However, she ${ }^{1}$ neither draws, nor expresses this relationship explicitly with a line. The rooms of the dormitory complex align the centrally positioned space along the main axis almost in the form of a cloister. The major public functions are situated at the two ends of the axis. It is clear that this regulating element provides the scaffolding around which the individual parts of the program have been organized.
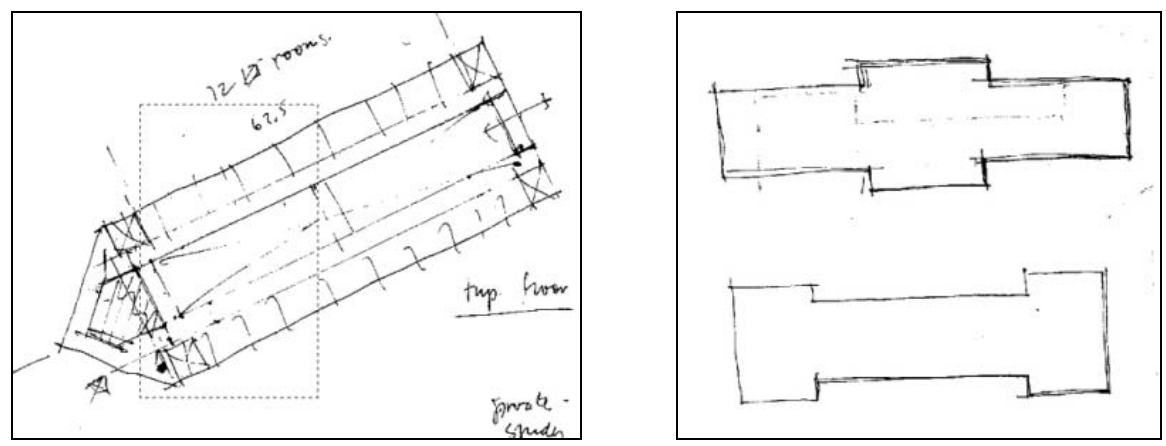

Figure 1: Implicit representation of axes of symmetry in S2 and S6, respectively.

This is not the only example of virtual axes elements used to define part whole relations in the protocols. In S6, for example (Figure 1), several axial, plan alternatives were developed, each with a different geometric structure. Yet, this participant does not express any explicit axes in his drawings.

\subsection{Organizing hierarchies}

The part-whole relationship naturally leads to the notion of hierarchy. Parts are subsumed by higher entities, which in turn define other, yet higher instances of design elements. It is not a surprise that architectural conventions include many examples of hierarchical decomposition: the building, its wings, their floors, suites found on floors, the individual rooms inside them, and so on. Both empirical evidence ${ }^{14}$ and normative models of design rely heavily on such spatial hierarchies ${ }^{15}$. In a widely published study Akin ${ }^{16}$ reports:

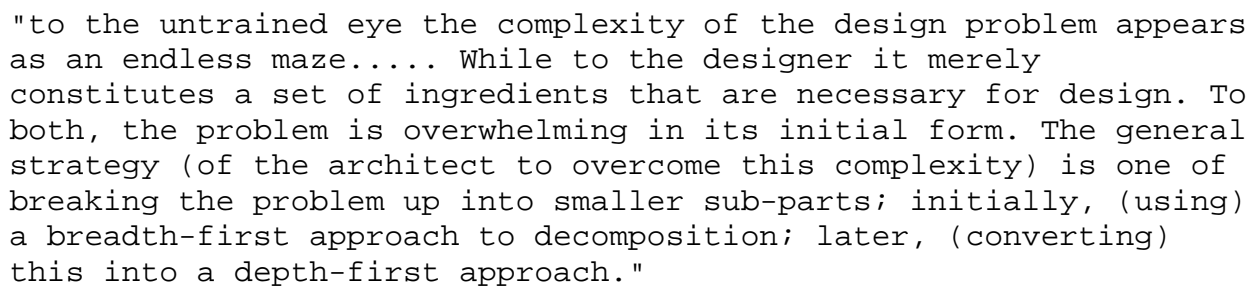

This peculiar combination of breadth-and-depth strategy is responsible for creating and navigating complex hierarchies in a design problem. First there is the decomposition of the problem into sub-parts. Since design problems do not arrive with predetermined (or a priori) sub-parts, the essence of the decomposition problem is to figure out a strategy for doing just that. Experienced designers have clearly developed approaches to accomplish this.

The evidence we gathered here suggests that massing, in fact, relies on these universally accepted strategies. The massing strategies defined here, which use regulating elements to create geometric order, establish a two-tier hierarchy between the regulator (super-node) and the regulated (sub-node). Nested regulating elements of massing then can create indefinitely deep hierarchies.

\footnotetext{
${ }^{1}$ For the purpose of anonymity, we consistently refer to second person singulars in the female gender. This is not for the purpose of signifying the gender of any individuals who may be referenced.
} 
In S4, the participant uses fence-stretch to lower the top vertex of two separate, but symmetrical roof elements. The participant wants to maintain the symmetry relation, yet she wants to adjust both roofs with a single step (Episode 3)

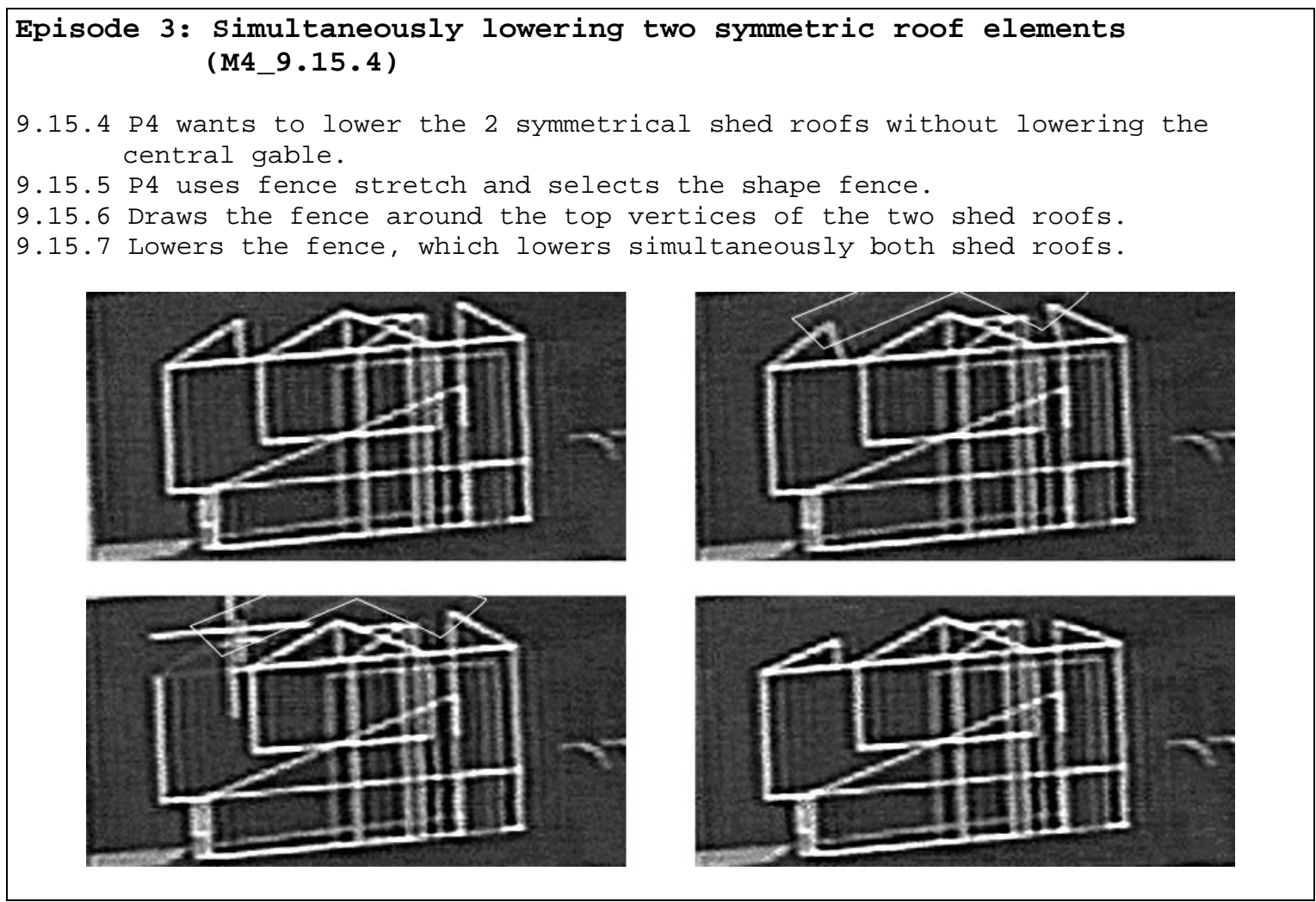

In S4, the participant also uses fence-stretch to manipulate two adjacent elements (Episode 4). Clearly, the bundling of the elements is governed by the parameters of the fence-stretch command of the drafting system being used. Conceptually, we can view this as an action that traverses two tiers of a multi-tiered element hierarchy.

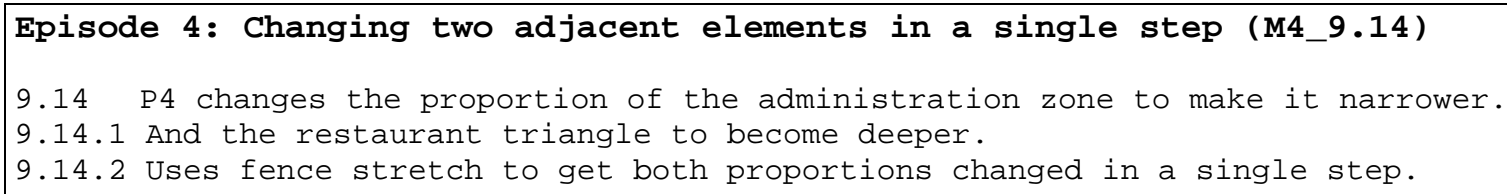

These examples also suggest a hierarchical relationship between elements, this time, however, between elements that are further down the ladder of hierarchy. These steps (9.14 and 9.15 in S4), seen together, suggest a hierarchy of elements that ascend from rooflines to room clusters to wings of buildings and, finally, to the entire building. The structure that govern such hierarchies have not been explained in design literature, other than to point to the part-whole relationship that exist between the immediate neighbors, that is, parent and child. When a regulating element is considered as part of the design hierarchy, it enables manipulations (Episode 3) that preserve the configuration's structure.

Why are some elements (for instance the floor of a wing) broken down into individual rooms, expressed by windows on the facade, while other elements of the same wing are represented through roof alignments between them and other wings of the building. Such complex hierarchies cannot be explained by simple spatial sub-sumption relationships like that of the spatial parents and children. However, they can be 
explained if regulating massing-elements are part of the hierarchy, each design element inheriting a property from a higher-level regulating element, such as an axis or alignment line.

\subsection{Scaffolding the design process}

Another view of our data relies on the scaffolding metaphor. Just as a scaffold provides a structure for accommodating construction activities, we observed that the relationship between the regulating elements and the spatial and physical massing of design elements rely on the framework created by the regulating elements. There appears to be a two-way interaction between the regulating and massing elements, particularly in the manner in which regulating elements are derived from masses and, in turn, masses are guided by regulating elements.

\subsubsection{By Extending Lines from Massing Elements}

Scaffold creation seems to be based on the extension of alignments in the current design. For instance, P4 extends a line from the boundary of an existing building on the given site, $\mathrm{MMCH}$, and uses it to align massing elements to preserve a consistent setback (Episode 5). The existing alignment serves as a scaffold for additional massing elements in the composition.

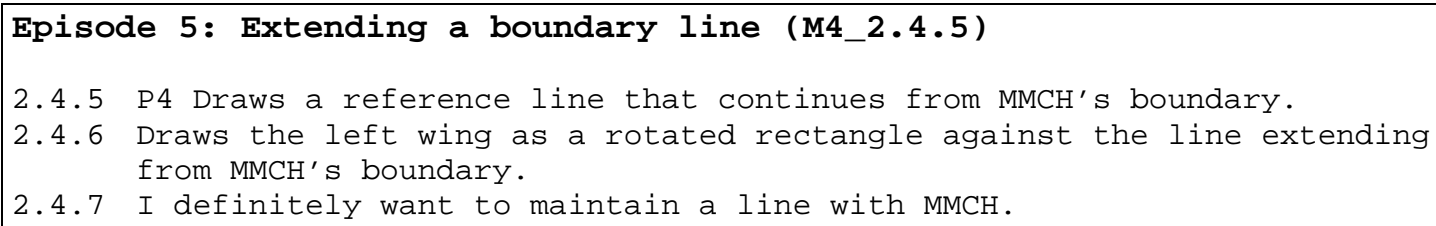

We observe $\mathrm{P} 4$ accomplishing similar ends using slightly different strategies. One of these is extending two lines from MMCH 's external protrusion and using them as guides to create a protrusion onto P4's proposed building. She then discovers a novel relation ${ }^{17}$ between these lines and her own buildings sub-structure, which causes an adjustment to the proposed massing configuration (Episode 6).

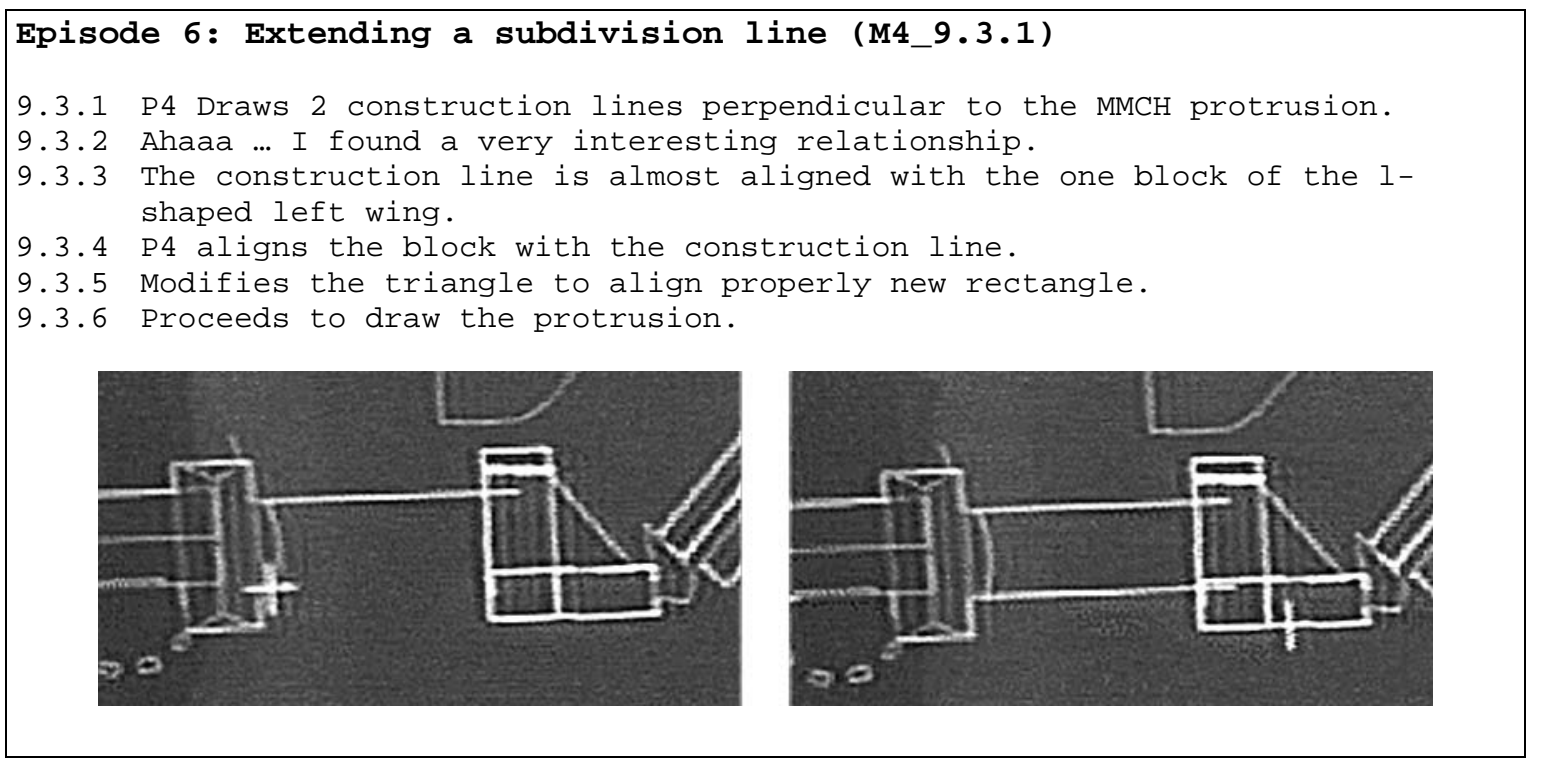

In another case of scaffolding, the new designs with existing elements, P1 draws a massing element, then derives its central axis as the major axis of the configuration, and then draws the other massing elements, organized by this axis. Rivka Oxman described semantic emergence as the emergence of the underlying 
structure or design organization ${ }^{18}$. In the following example, $\mathrm{P} 1$ has recognized the emergence of the central organizing axis, in the middle of the process of creating massing elements. This is significant because it shows that emergent features (implicit axes) are just as likely to become scaffolds as explicit ones (Episode 7).



\subsubsection{By Extending Plan and Elevation Lines}

Finally, we observe P3 using a strategy that also falls in the category of scaffolding. P3 derives the geometric structure of the elevations, by extending the axes of the plan, thus reusing the regulating elements of the plan in the elevation (Episode 8).

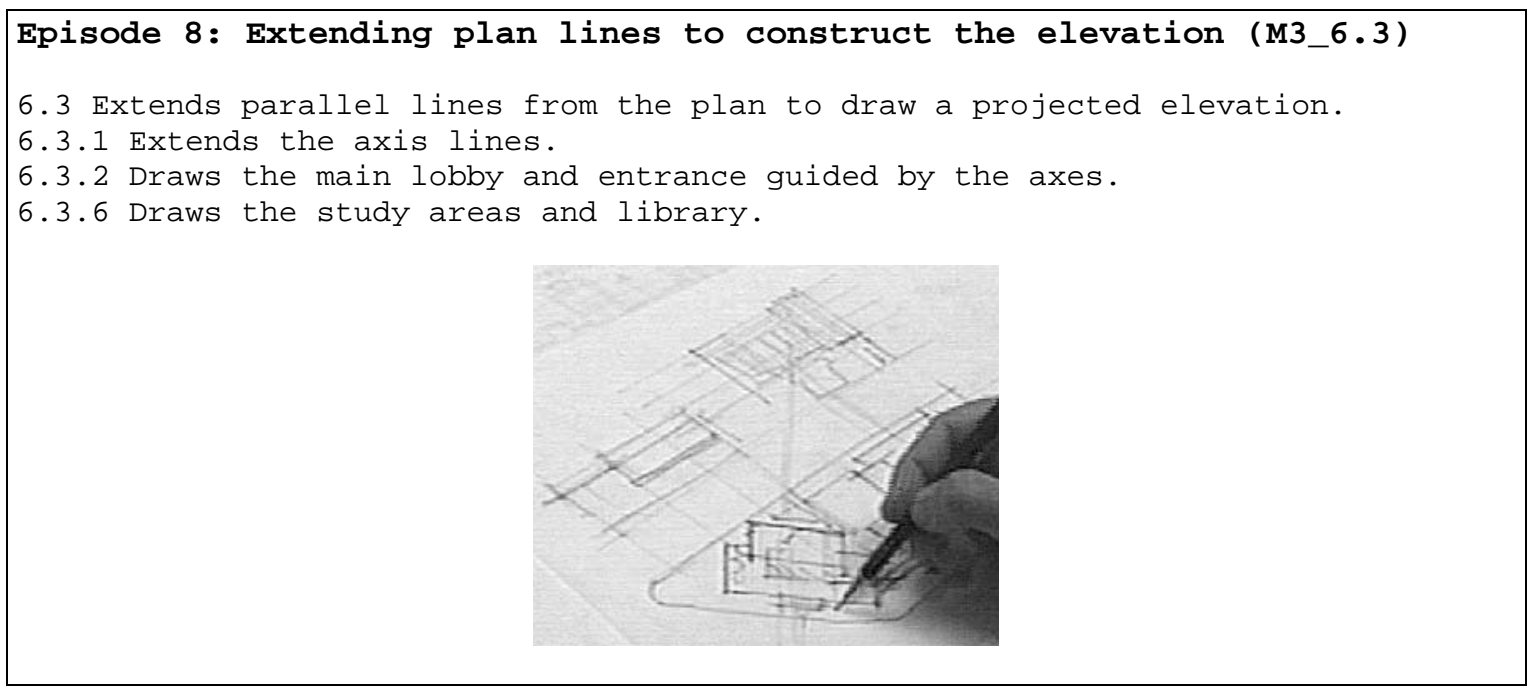

\subsection{Topology and Geometry}

Topology captures adjacency relationships within a configuration. March discussed topological relationships extensively. He illustrated the notion with three of Frank Lloyd Wright houses Life, Jester and Sundt, which share the same topological structure, exemplified by the same programmatic relations, while their individually geometries being radically different (Figure 2). ${ }^{19}$ 


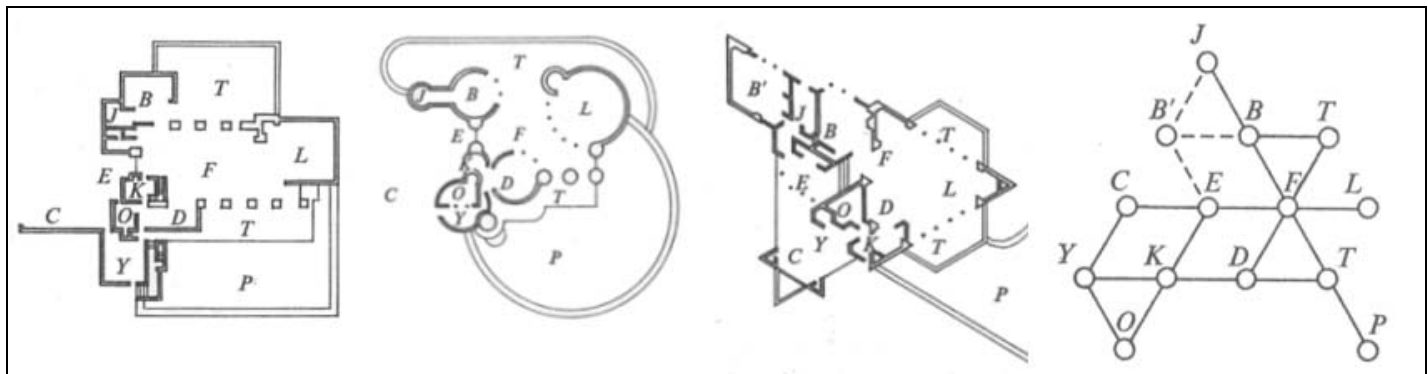

Figure 2: Frank Lloyd Wright houses (Life, Jester and Sundt) with identical topology and variable geometry: Source March 1970

Regulating elements of massing also appear to be one of the rare design tools that are suitable for representing the typology of a given geometric composition. For instance, axes can be used to represent associations and alignments of spaces independent of shape and size. Spaces can be strung along an axis creating a linear topological structure. Alternatively, multiple axes (either representing symmetry or alignment) can be used to create much more complex relationships, like grids and urban road patterns, once again, to preserve the topological relationship between the elements whether they are rooms or city blocks.

\subsubsection{From the Context}

The topological structure of a massing configuration can be defined in several ways. These styles are not mutually exclusive, and occur in hybrid combinations. A primary strategy we found in our data is the creation of topological relationships from contextual information provided by axes. For instance, P2 starts his design by deriving the geometric structure from the site (Episode 9). She then picks up the axis of the streets and the setbacks from a neighboring building, $\mathrm{MMCH}$.

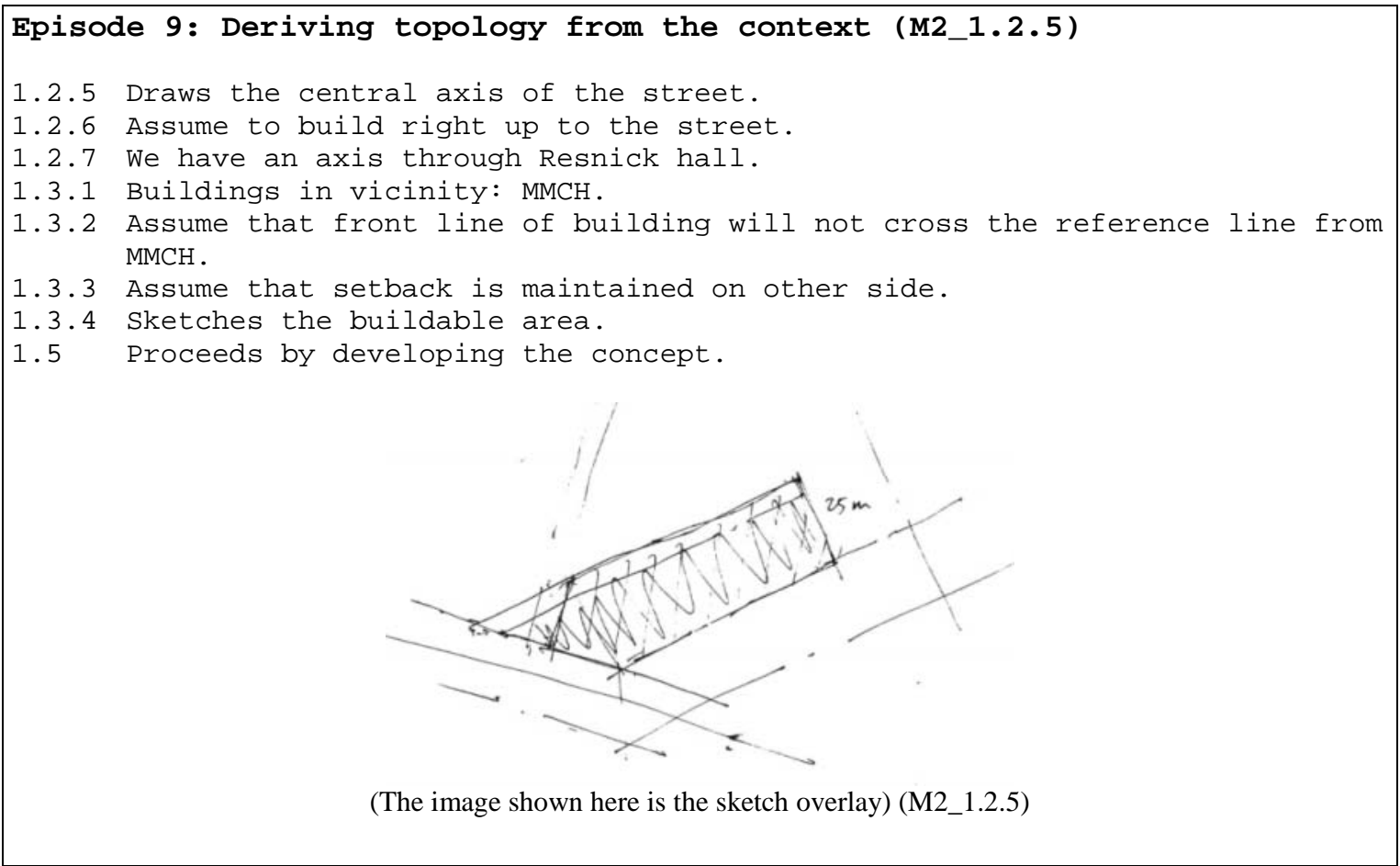




\subsubsection{From program relations}

We also observed that similar topological structures emerge from the building program. P1 and P3, for example, determine the initial geometric structure of their designs from the spatial relations included in the program. In moves illustrated in Episode 10, P1 expresses the topological structure of the program, even before creating graphic representations of the design.

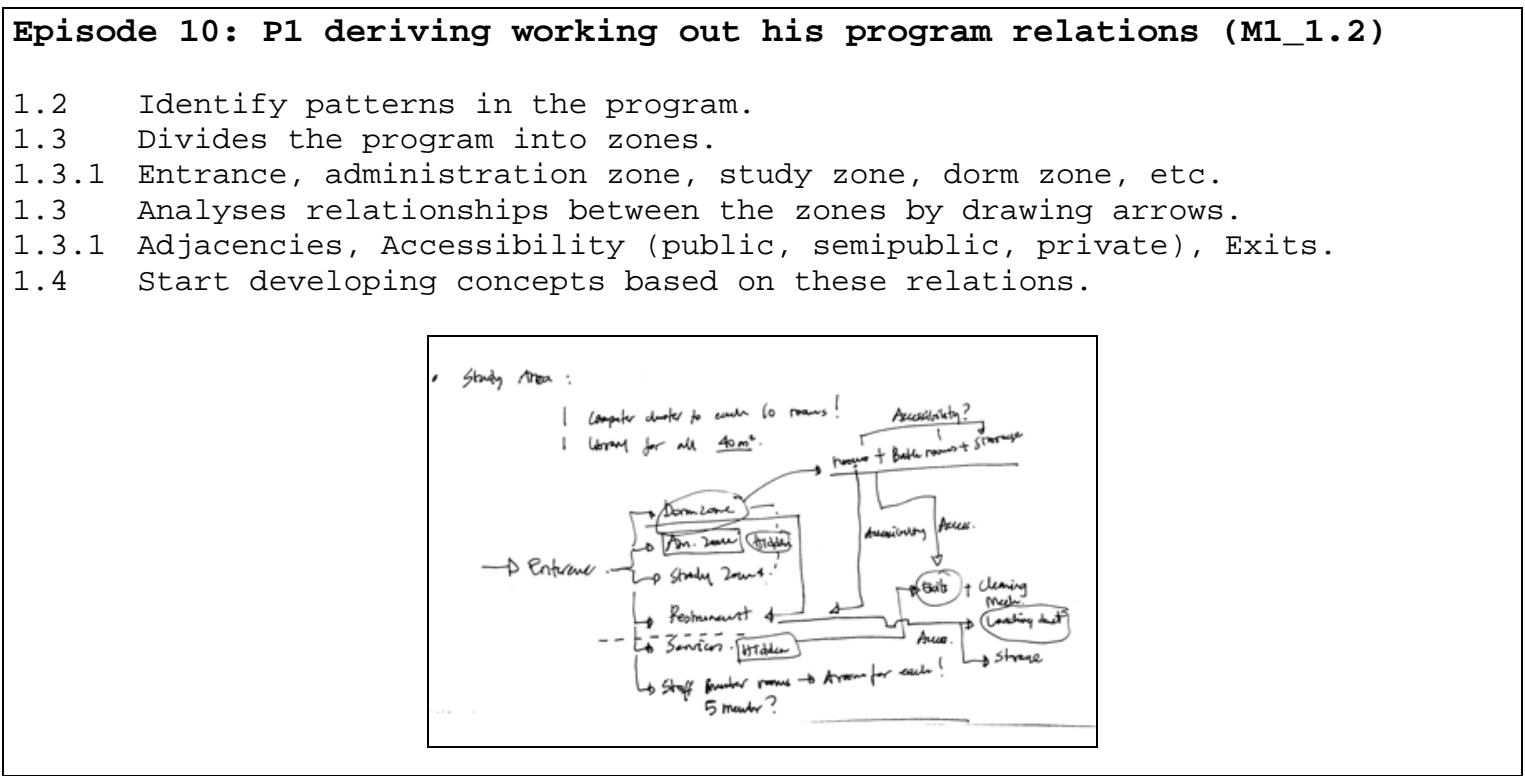

\subsection{Structuring the ill-structured design task}

Simon's cognitive model describes the design process as problem solving and categorizes design problems as ill-structured. Design is considered as a search for an adequate solution within a large space of alternative ${ }^{20}$. Akin explains that problem structuring is a prerequisite to problem solving; problem structuring consists of a series of transformations converting an ill-structured problem to a well-structured $o^{21}{ }^{21}$. In this initial phase, design specifications and relations that need to be satisfied in the design solution are defined. Failure to satisfy these would lead to restructuring of the problem, which takes the form of modifying relations and redefining specifications, thus transforming the problem by altering its parameters. In other words, the solution reconstructed according to the new problem parameters.

Another relevant paradigm, introduced by Archea, refers to design structuring as puzzle making ${ }^{22}$. Maher has introduced a different form of restructuring through the co-evolutionary model for design exploration ${ }^{23}$. This model, which is motivated by the fact that designers explore the problem space as well as the solution spaces, supports the simultaneous evolution of problems and solutions by means of genetic algorithms.

\subsubsection{Structuring the problem}

One of the most straightforward ways of structuring an ill-structured problem is to break it into more and more specialized parts. Massing with regulating elements seems to achieve this as well. In this case, the decomposition of the design problem is graphically driven.

Our participants appear to create local problem sub-structures by adding sub-division lines into the massing representation. For example, P1 uses modular subdivisions lines to develop the sub-structure of the configuration (Episode 11). She then uses these as guides in protruding and recessing parts of the mass. 


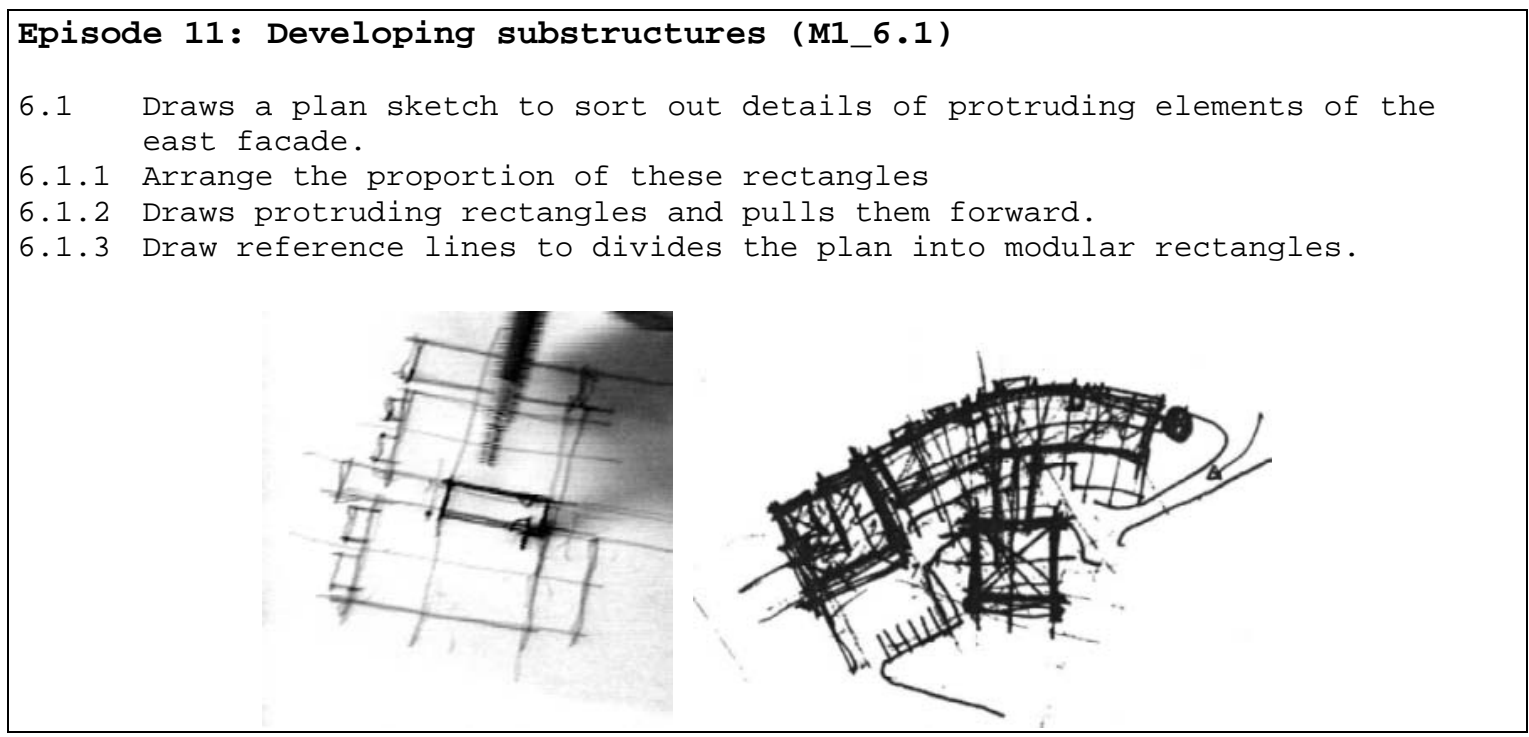

Episode 12: Developing the structure by subdividing the mass (M2_4.2.3)
4.2.3 Subdivide to show the rooms.
4.2.4 There might be a community thing at the pointed end.
4.2.5 There might be a bridge half way down.
4.2.6 The entrance would be from here, and shows slanted side.
4.2.7 There will be fire stairs at the four corners of the rectangle.

In another example, $\mathrm{P} 2$ uses subdivisions to derive the internal geometric structure of the building (Episode 12). In a slightly different case, $P 3$ develops local structures and re-uses the main geometric structure of the massing configuration (Episode 13) in an attempt to elaborate his configuration in greater detail. This is a clear example of the local feature inheriting structure of the overall configuration.






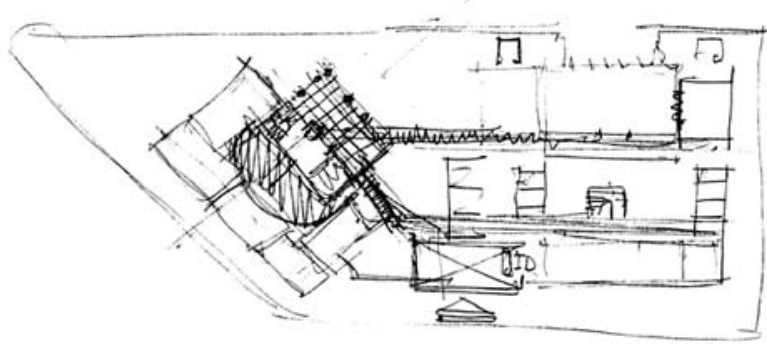

\subsubsection{Problem Restructuring}

One of the behavioral characteristics of expert designers is a skill and propensity to restructure design problems (Akin, et al., 1987). In our data, we encountered several forms of this in the context of massing through regulating elements.

A frequent form of this is through the development of alternatives. For example, P6 developed six alternatives (five manual and one computer based) to visualize different geometric structures (Figure 3).

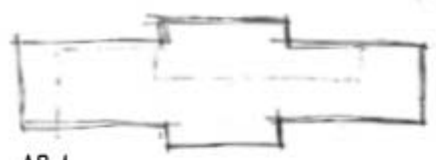

A6_

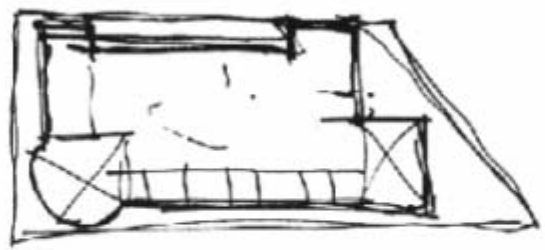

A6 4

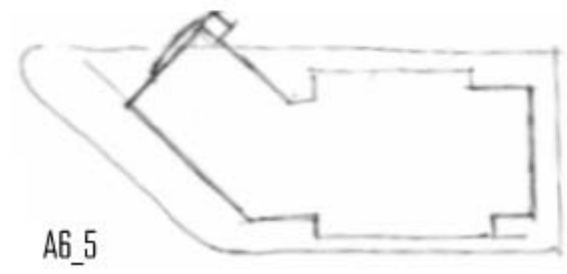



A6_2



A6_3

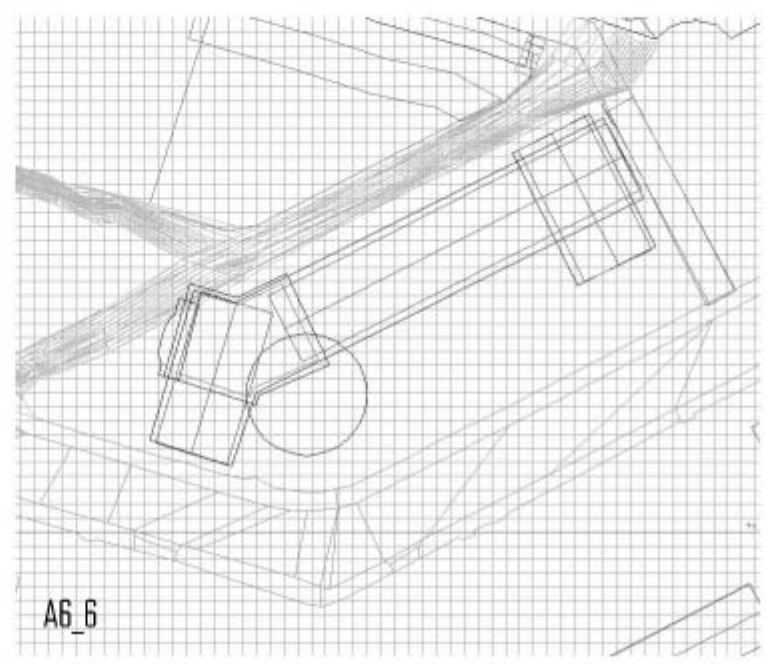

A6_1 has one main central axis and a secondary perpendicular axis in the middle.

A6_2 has one main axis and two secondary axes on the edges.

A6_3 is a variation of A6_2, but has a section subtracted to break the symmetry.

A6_4 a rectangular structure with no dominating axes.

A6_5 had an axis bent to 45 degrees.

A6_6 computer alternative combining axes of A6_2, bending concept of A6_5, and a circular element

Figure 3: P6's Alternative illustrating a major change in structure.

Moving from A6_1 to A6_2 is a shift in the central secondary axis and an addition of another axis. Moving from A6_1 to A6_5 is a bent in the longitudinal axis, and to A6_6 is a bend in the other direction. 
Similarly, P1 develops three alternative configurations. Moving from A1_1 to A1_2 is a major change in ordering principle, the symmetry and well as the homogeneity is broken, new concepts emerge and new local grids are added (Figure 4). Moving from A1_2 to A1_3 however, is less dramatic, since the bridge concept is in both configurations, as well as the movement along the curved path, and can be summarized as breaking the large curvilinear mass into separate components, with different forms and orientations.

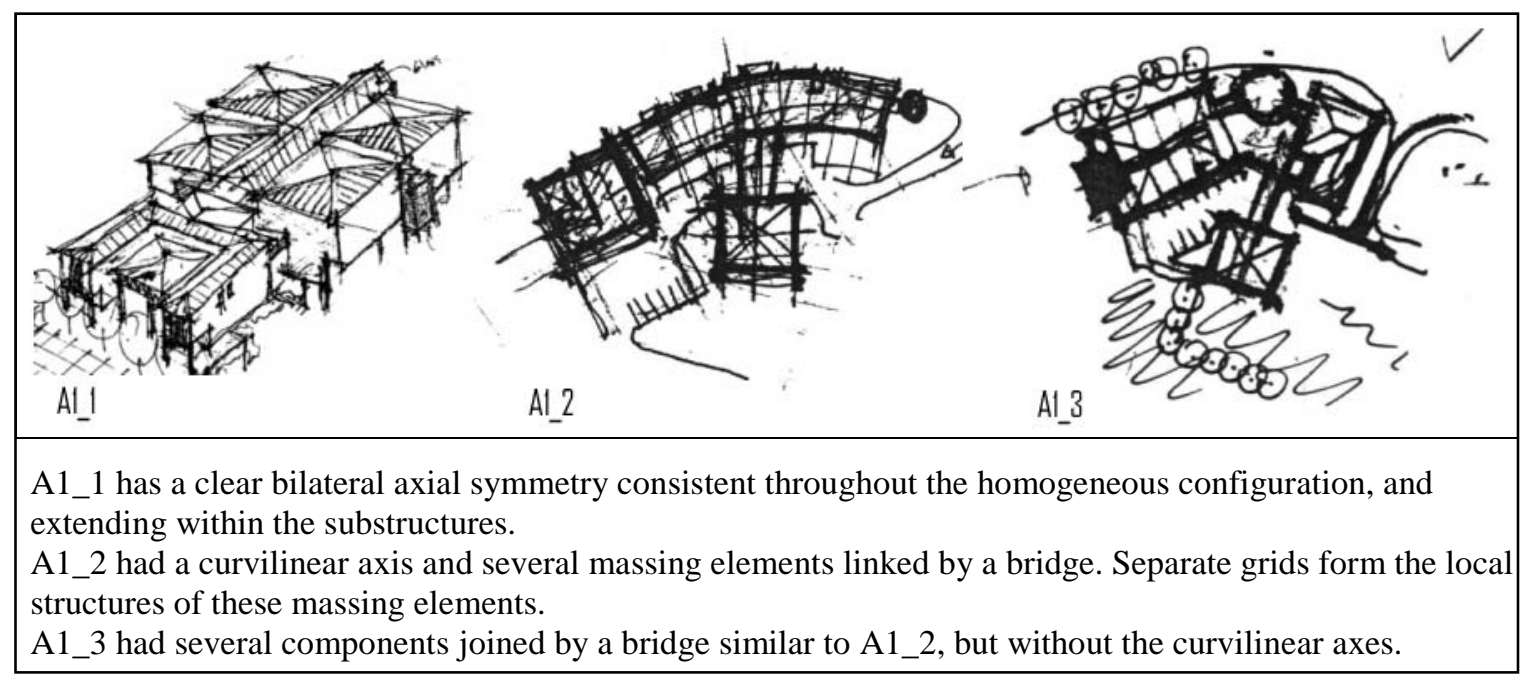

Figure 4: P1's alternatives illustrating major changes in structure

Restructuring the problem does not always mean a wholesale redesign or the generation of an entirely new alternative. Occasionally, we observed our participants achieving the same effect by modifying key elements in the solution domain. For example, P4 draws his roof configuration on paper. P4 starts by drawing the right wing, following its geometric structure, then draws that of the left wing. P4 starts by following the left wing's geometric structure, but then gets an idea (at this point P4 asks for tracing paper), and redraws over his configuration and rotates the axis of the roof by 90 degrees. Then $\mathrm{P} 4$ draws two symmetrical gables parallel to the new axis, partially restructuring the roof configuration (Episode 14).







Alternatively, P1 engages in restructuring as a means of developing a single alternative. P1 changes the curvature of his building, from curvilinear to rectilinear, which illustrates a major restructuring within the same alternative (Episode 15). 
Finally, we note that P1 creates two variations of the third alternative, where the rotated the axes of the right wing, changed the proportions of the right wing and shifted the bridge's axis (Episode 16).



\section{Discussion}

The protocols results illustrated several situations where designers handled the geometric structure of a massing configuration in such a way that they seemed to be doing more than just composing forms. Repeatedly and consistently, we found behaviors that structure and manage the design development process.

It is our contention that this is not merely a coincidence. We believe designers use the medium of representing massing elements, and in particular, elements of regulation to mange the design process itself. We showed this behavior in many guises related to design literature that deals with descriptive views of design. This includes management of part-whole relationships, design hierarchy, topology-geometry relationships, scaffolding the design process, structuring ill-structured problems, and the restructuring of problem parameters. Each one of these is a separate facet of the same phenomenon of managing the design process through massing representations.

We would like to conclude our analysis by discussing computational support for these strategies.

A major issue of concern is to capture the massing configuration's structure by means of regulating elements in the underlying computational representation and massing strategies that go with them. These strategies can be enhanced even further, if we assume that the regulating elements could be flexible entities. By interactively manipulating them, the designer could propagate changes onto the regulated elements.

There have been very few attempts to utilize regulating lines as means for manipulation in CAD representations. Branko Kolarevic addressed alignment and grid structures as regulating concepts. His approach has been based on the drafting metaphor, where pencil (or construction) lines regulated ink (or final design) lines. ${ }^{24}$ In an earlier prototype, we addressed symmetry structures as a regulating concept. Centers of rotation and axes of mirror, translation, and glide, are treated as regulating elements that have control over other objects. ${ }^{25}$ This system supports many of the protocol segments illustrated in this paper such as P4's strategy of simultaneously manipulating the symmetric roof structure, which can be done by using a mirror regulating element.

We would like to extend these efforts by supporting additional operations such as subdividing, which is a key tool for structuring strategies. A designer would then structure her configuration, by subdivision and decomposition, and can develop her designs thought manipulations that preserve this structure. 
Regulating elements thought decomposition would contribute to hierarchic design strategies. Regulating elements could then become instruments to control massing elements by propagating changes throughout the hierarchy.

Another major motivation is to support the two-way interaction among massing elements and regulating elements observed in the scaffolding category. Regulators can be automatically derived from massing elements by extension of axes, boundaries or subdivisions. Additionally, we would like to support the twoway interaction between topology and geometry. A designer can manipulate the spaces specified by the program and assign relations, then derive masses. Alternatively, she can define masses then insert the spaces in those masses.

The ability to restructure massing configurations and instantaneously observe their complete effects is perhaps the most exciting and rewarding aspect of such a computational tool. Verstijnen, et.al. (2002) reported that the act of restructuring is difficult to conceive with mental imagery alone, and is significantly enhanced by sketching. ${ }^{26}$ They also conducted studies illustrating that current computing tools do not support design-restructuring behavior. It is our intention to show that with the help of interactive regulating elements, the typically time-consuming labor-intensive act of restructuring can be carried out instantly and accurately in a computational environment.

We consider that an exploration tool for supporting these strategies, which is underway, will greatly enhance massing design process and will be a significant step forward in CAD research. 


\section{Appendix 1: Experiment instructions}

This is a protocol experiment designed to explore the way architects design the three-dimensional configuration of buildings. Your task is to design a building and produce a 3-D drawing that illustrates your design.

\section{The design problem:}

The university needs additional student housing facilities. Since the accommodations in Donner Hall are not satisfactory, the university has decided to take it down and replace it with a new, satisfactory housing facility for students. The facility will provide accommodations for 50 graduate students, 150 undergraduate students, and 5 staff members. This building is intended to serve as a model for housing facilities that other colleges would follow. The site plan and the architectural program are provided on the following pages.

\section{The task:}

Your task is to design the 3-D configuration of this facility; you are encouraged to make an outstanding design that will be the object of admiration of all the Carnegie Mellon community.

\section{Requirements for the sketch-based experiment}

The requirements for this experiment is to draw the 3-D model, as well as the corresponding plan layouts, sections or elevations, on the provided transparent sketch- pad. Feel free to use overlays, as you explore solutions and modify them.

- For the 3-D model, you may draw an axonometric, isometric or perspective.

- Please speak your mind as you design.

- You may take as much time as you need.

- Please feel free to ask questions at any time during the experiment.

\section{Requirements for the computer-based experiment}

The task is to design a building, while using a computer and generate a model that illustrates your design. you. A computer model of the site is located in the file expB.

For this experiment, you will use the CAD system provided (FormZ, AutoCAD, or Microstation) to design your building. You are free to use the provided site model as a base for your design or to start your design from a new CAD file. If you do choose to use the provided expB file, please do not modify the site model.

- Please speak your mind as you design

- You may take as much time as you need.

- Please feel free to ask questions at any time during the experiment. 


\section{Appendix 2: Questionnaire \\ Common questions for the sketch based and computer based experiments}

Architectural Education:

Years experience in practicing Architectural Design:

Years experience in teaching Architectural Design.:

What is your preferred CAD system?

Years Experience with your preferred CAD system:

Do you have experience with other CAD system? (Please list):

For what purpose do you usually use a CAD system?

- drafting $\bullet$ constructing design ideas $\bullet$ conceptual design $\bullet$ others:

Were you able to modify your design freely and try out several configurations?

Did you feel you needed more information to perform the task? If so what type of information was missing?

Was there any action/task that you wanted to do and could not do with the sketch medium. If so, which ones?

Is there any other activity that you typically do when you are designing the 3-D configuration of building? Please list.

If you had a computer tool that specializes in 3-D building configuration design which features would you have liked it to have? Please explain.

\section{Additional questions the computer-based experiment}

Were any of the tools used in this experiment limiting in any way? Please explain.

Are you familiar with any other computer 3-D modeler? If yes which ones?

Which computer 3-D modeler do you prefer? Why?

If you were to add a new design tool to the provided CAD tool, what would it be? 


\section{Appendix 3: Architectural program for a dormitory facility on the Carnegie Mellon campus}

\begin{tabular}{|c|c|c|c|c|c|}
\hline \multirow[t]{2}{*}{ Space } & \multirow[t]{2}{*}{\begin{tabular}{|l|} 
No of \\
Spaces
\end{tabular}} & \multirow{2}{*}{$\begin{array}{l}\text { Size per } \\
\text { space } \\
\mathrm{m}^{2}\end{array}$} & \multirow[b]{2}{*}{$\mathrm{ft}^{2}$} & \multicolumn{2}{|c|}{ Total Size } \\
\hline & & & & $\mathrm{m}^{2}$ & $\mathrm{ft}^{2}$ \\
\hline \multicolumn{6}{|l|}{ Private and Semi private } \\
\hline Student rooms & 100 & 20 & 220 & 2000 & 22000 \\
\hline Bathrooms & 25 & 8 & 90 & 200 & 2250 \\
\hline Storage rooms & 25 & 8 & 90 & 200 & 2250 \\
\hline Lounges with kitchenettes & 25 & 20 & 220 & 500 & 5500 \\
\hline Laundry rooms & 10 & 10 & 110 & 100 & 1100 \\
\hline \multicolumn{6}{|l|}{ Study areas } \\
\hline Computer clusters & 10 & 20 & 220 & 200 & 2200 \\
\hline Library and study area & 1 & 40 & 430 & 40 & 430 \\
\hline Main Lobby. & 1 & 30 & 320 & 30 & 320 \\
\hline Administration area. & & & & & \\
\hline Social counseling & 5 & 12 & 130 & 60 & 650 \\
\hline Meeting room & 1 & 12 & 130 & 12 & 130 \\
\hline \multicolumn{6}{|l|}{ Restaurant. } \\
\hline Kitchen & 1 & 40 & 430 & 40 & 430 \\
\hline Dining area & 4 & 50 & 540 & 200 & 2160 \\
\hline \multicolumn{6}{|l|}{ Support Spaces } \\
\hline Emergency exits & 4 & 10 & 110 & 40 & 440 \\
\hline Cleaning storage spaces & 4 & 12 & 130 & 48 & 520 \\
\hline Mechanical rooms & 1 & 50 & 540 & 50 & 540 \\
\hline Loading dock & 1 & 50 & 540 & 50 & 540 \\
\hline Loading elevator & 1 & 10 & 110 & 10 & 110 \\
\hline Storage & 1 & 30 & 320 & 30 & 320 \\
\hline Total & & & & 3810 & 41890 \\
\hline Circulation. & $20 \%$ & & & 760 & 8380 \\
\hline
\end{tabular}




\section{Appendix 4: Site}

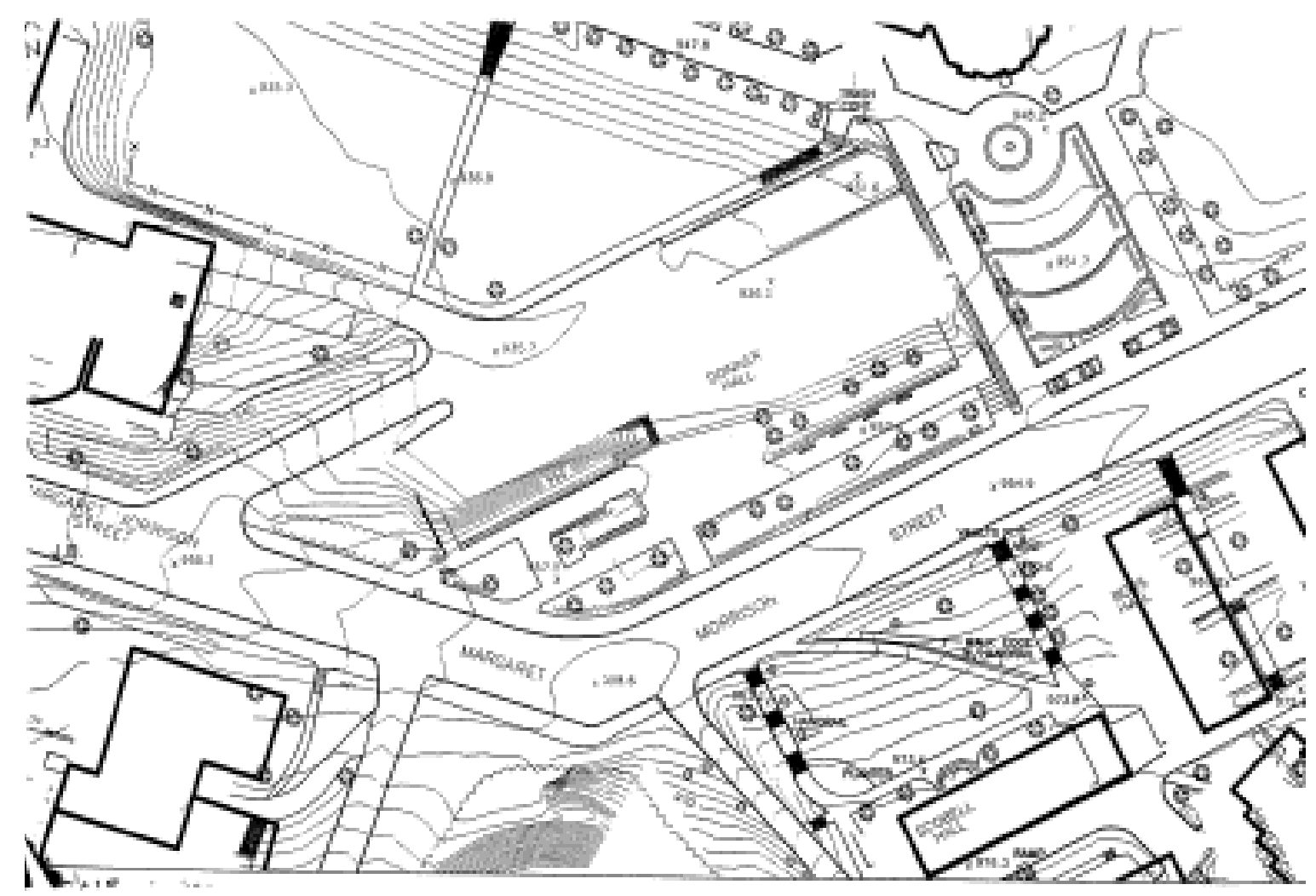




\section{References}

${ }^{1}$ Le Corbusier. Towards a new Architecture. Preager Publishers inc., 1960 .p31.

${ }^{2}$ Kirwan, B., Ainsworth, L. A Guide to Task Analysis. Taylor and Francis, London, . 1993.

${ }^{3}$ Ericsson, K., Simon, H. Protocol Analysis: Verbal Reports as Data, MIT Press, Cambridge, Mass., 1993.

${ }^{4}$ Moustapha, H. Architectural Explorations: Transforming Configurations through Interactive Design Structures. Carnegie Mellon University, Pittsburgh 2001.

${ }^{5}$ Chimacoff, A. Figure, System and Memory: The Process of Design. In Representation and Architecture. Information Dynamics Inc., Silver Springs, 1992.

${ }^{6}$ Le Corbusier. Towards a new Architecture. Preager Publishers inc., 1960 . pg71

${ }^{7}$ Madrazo, L. Durant and The Science of Architecture. In the Journal of Architectural Education, vol. 48/1. Association of Collegiate Schools of Architecture. Washington, May 1994. p17

${ }^{8}$ Akin, O. How Architects Design. Artificial Intelligence and Pattern Recognition in Design, North Holland Publishing Company, Amsterdam, 1978.

${ }^{9}$ Akin, O. Psychology of Early Design. Technical Report, Carnegie Mellon University, Pittsburgh, 1994.

${ }^{10}$ Larkin, J., Simon H. Why a Diagram is Worth Ten Thousand Words. Cognitive Science Journal vol. 11. , 1987.

${ }^{11}$ Suwa, M. Tversky, B. What Architects and Students See in Architectural Design Sketches? in Descriptive Models of Design, Istanbul 1996.

${ }^{12}$ Van Sommers. P. Drawings and Cognition: Descriptive and Experimental Studies of Graphics Production Processes. Cambridge University Press, Cambridge, 1984.

${ }^{13}$ Goldschmidt, G. On Visual Design Thinking: The Wiz Kids of Architecture. Design Studies, vol. 15 \#2, Butterworth and Co. Ltd. 1990.

${ }^{14}$ Eastman, C. Cognitive Processes and Ill-defined problems, Proceedings of the International Joint Conference on Artificial Intelligence, 1969.

${ }^{15}$ Flemming U. Woodbury R. Software Environment to support early phases of in building design SEED Overview. In Journal of Architectural Engineering, vol. 1, no. 4, American Society of Civil Engineers. Architectural Engineering Division, 1995.

${ }^{16}$ Akin, O., Akin, C. On the process of creativity in puzzles, inventions and designs in Automation in Construction,. "Editorial" (Special issue: models of design) Automation in Construction, 7 (1998).

${ }^{17}$ Akin, O. Akin, C. Expertise and Creativity in Architectural Design. Descriptive Models of Design, Istanbul, 1996.

${ }^{18}$ Oxman, R., The Thinking Eye: Visual recognition in Design Emergence. Design studies, Elsevier Science ltd, London, England. 2002.

${ }^{19}$ March, L., Steadman P. The Geometry of the Environment . The MIT press, Cambridge Massachusetts, 1974.

${ }^{20}$ Simon H. The Science of the Artificial. MIT press, Cambridge, Mass, 1969.

${ }^{21}$ Akin, O. Problem Structuring in Architectural Design . Technical Report, Carnegie Mellon University, Pittsburgh, 1987.

${ }^{22}$ Archea, J. Puzzle Making: What Architects Do When No One is Looking . In Computability of Design. Editor: Kalay Y. John Wiley and Sons Inc., NY, 1987.

${ }^{23}$ Maher, M,. Modelling Design Exploration as Co-Evolution, Microcomputers in Civil Engineering, 1996.

${ }^{24}$ Kolarevic, B. Lines And Geometric Relations as a Framework for Exploring Shape, Dimension and Geometric Organization in Design . In CAAD Futures 97, Kluwer Academic Publishers, Munich,1997 
${ }^{25}$ Moustapha, H., Krishnamurti, R. Arabic Calligraphy: A Computational Exploration. in Mathematics and Design 2001. Third International Conference, Geelong 2001.

${ }^{26}$ Vertijnen et al, Sketching and Creative Discovery Design studies, Elsevier Science ltd, London, England. 2002. 